\title{
Fgf20 governs formation of primary and secondary dermal condensations in developing hair follicles
}

\author{
Sung-Ho Huh, ${ }^{1,3}$ Katja Närhi, ${ }^{2,3}$ Päivi H. Lindfors, ${ }^{2}$ Otso Häärä, ${ }^{2}$ Lu Yang, ${ }^{1}$ David M. Ornitz, ${ }^{1,4}$ \\ and Marja L. Mikkola ${ }^{2,4}$ \\ ${ }^{1}$ Department of Developmental Biology, Washington University School of Medicine, St. Louis, Missouri 63110, USA; \\ ${ }^{2}$ Developmental Biology Program, Institute of Biotechnology, University of Helsinki, 00014 Helsinki, Finland
}

\begin{abstract}
In hair follicle development, a placode-derived signal is believed to induce formation of the dermal condensation, an essential component of ectodermal organs. However, the identity of this signal is unknown. Furthermore, although induction and patterning of hair follicles are intimately linked, it is not known whether the mesenchymal condensation is necessary for inducing the initial epithelial pattern. Here, we show that fibroblast growth factor $20(F g f 20)$ is expressed in hair placodes and is induced by and functions downstream from epithelial ectodysplasin (Eda)/Edar and Wnt/ $\beta$-Catenin signaling to initiate formation of the underlying dermal condensation. Fgf20 governs formation of primary and secondary dermal condensations in developing hair follicles and subsequent formation of guard, awl, and auchene hairs. Although primary dermal condensations are absent in Fgf20 mutant mice, a regular array of hair placodes is formed, demonstrating that the epithelial patterning process is independent of known histological and molecular markers of underlying mesenchymal patterns during the initial stages of hair follicle development.
\end{abstract}

[Keywords: Eda signal; Fgf20; Wnt signal; dermal condensation initiation; hair follicle development; mesenchymalepithelial interactions]

Supplemental material is available for this article.

Received June 19, 2012; revised version accepted January 10, 2013.

Reciprocal mesenchymal and epithelial interactions are essential for the development of skin appendages, such as teeth, mammary glands, and hair (Schmidt-Ullrich and Paus 2005; Mikkola and Millar 2006; Fuchs 2007). Induction of hair follicle development is marked by reorganization of basal keratinocytes into a placode and clustering of underlying mesenchymal cells into a condensation, the precursor of the dermal papilla. The earliest known event in hair follicle development involves activation of epithelial Wnt/ $\beta$-Catenin signaling: Its absence prevents (Zhang et al. 2009), and its forced activation advances (Närhi et al. 2008; Zhang et al. 2008), hair follicle induction. Subsequently, epithelial ectodysplasin (Eda)/Edar signaling is required to refine and maintain the pattern of Wnt/ $\beta$-Catenin activity to enable primary hair placode formation (Mou et al. 2006; Fliniaux et al. 2008; Zhang et al. 2009). Pioneering tissue recombination experiments suggest that induction of hair follicles depends

\footnotetext{
${ }^{3}$ These authors contributed equally to this work.

${ }^{4}$ Corresponding authors

E-mail dornitz@wustl.edu

E-mail marja.mikkola@helsinki.fi

Article is online at http://www.genesdev.org/cgi/doi/10.1101/gad.198945.112.
}

on unknown cues originating from the dermis that are required for the formation of placodes (Kollar 1970; Dhouailly 1973; Hardy 1992). It is thought that a placodederived signal subsequently induces condensation of underlying dermal cells, but the identity of this signal has been elusive (Dhouailly 1973). Although induction and patterning of hair follicles are tightly coupled (Andl et al. 2002; Sick et al. 2006; Zhang et al. 2009; Sennett and Rendl 2012), it is not known whether mesenchymal condensation or a mesenchymal prepattern is necessary for hair placode patterning.

Fibroblast growth factor (Fgf) signals function during multiple stages of hair follicle development. Fgf receptor $2 b($ Fgfr $2 b)$ is expressed in the epithelium of developing skin, and deletion of Fgfr2b resulted in fewer and retarded hair follicle formation (Petiot et al. 2003; Richardson et al. 2009). In addition, deletion of both Fgfr1 and Fgfr2 in keratinocytes resulted in progressive loss of hair follicles in adult mice (Yang et al. 2010). Fgf10 is required for whisker formation, whereas Fgf7 is suggested to inhibit hair follicle induction (Ohuchi et al. 2003; Richardson et al. 2009).

Here, we identify an essential role for Fgf2O in the induction of dermal condensations in primary and secondary 
hair follicles. These studies also lead to the unexpected conclusion that epithelial and mesenchymal morphogenesis can be uncoupled during the initial steps of hair follicle development.

\section{Results}

Canonical Eda/Edar and Wnt/ $\beta$-Catenin signals regulate Fgf20 expression in vivo and in vitro

While screening for epithelial factors that are regulated by the Eda/Edar pathway, we identified Fgf2O as a gene rapidly induced following treatment of $E d a^{-/-}$(Tabby mutant) skin explants with recombinant EDA (Lefebvre et al. 2012). The Fgf20 message was increased 3.3-fold and 16-fold following treatment with EDA for 2 and $4 \mathrm{~h}$, respectively, compared with untreated controls (Fig. 1A). Analysis of Fgf20 expression using an Fgf20- $\beta$-galactosidase ( $\beta$ Gal) knock-in allele (Fgf20 ${ }^{\beta G a l}$ ) (Huh et al. 2012) revealed focal $\mathrm{Fgf20}$ expression as early as embryonic day E13.5 (E13.5), prior to the appearance of morphologically distinct primary hair placodes (Fig. 1B; Supplemental Fig. S1A). By E14.5, Fgf20 ${ }^{\beta G a l}$ expression was prominent in the placodal epithelium in a pattern that matched Edar (Laurikkala et al. 2002) and Fgf20 expression, as determined
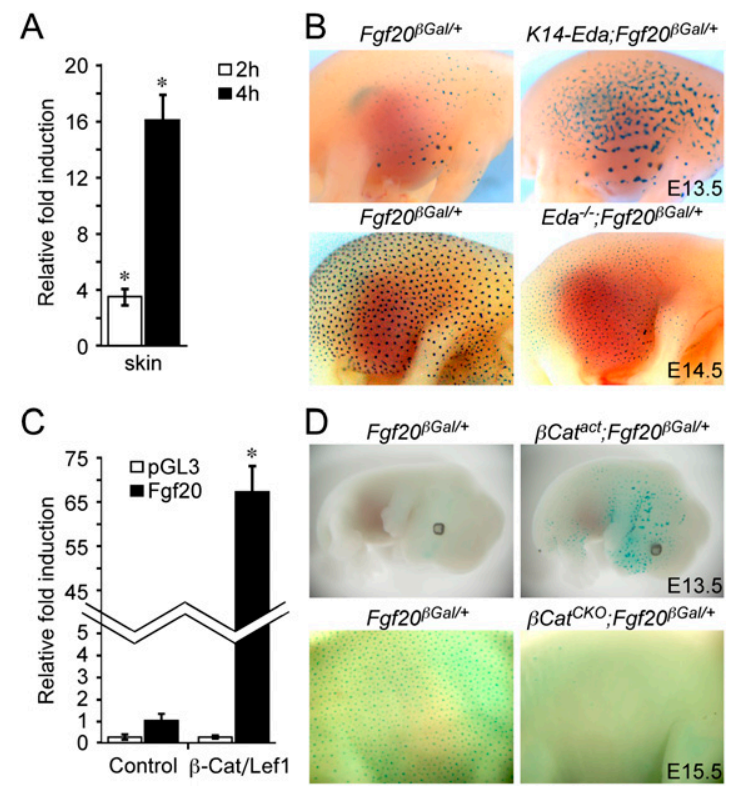

Figure 1. Fgf2O is a target of Eda/Edar and Wnt/ $\beta$-Catenin signal during hair placode formation. (A) Real-time quantitative PCR (qPCR) for Fgf20 from E14.5 Eda ${ }^{-1-}$ skin explants with or without EDA treatment for $2 \mathrm{~h}(n=12 ; P=0.008)$ or $4 \mathrm{~h}(n=6$; $P=0.03)$. Data are shown as mean \pm SD. $(B) \beta$ Gal staining indicating Fgf $20^{\beta \mathrm{Gal}}$ expression in K14-Eda;Fgf20 ${ }^{\beta G a l /+}$ and $E d a^{-/} ; F g f 20^{\beta G a l /+}$ embryos. (C) Assay showing that a $5.2-\mathrm{kb}$ genomic DNA fragment upstream of the Fgf2O transcription initiation site driving luciferase was induced by transfection of a $\beta$-Catenin-Lef 1 fusion protein $(n=9 ; P<0.001)$. The vector control pGL3 showed no induction. Data are shown as mean \pm SD. $(D) \beta \mathrm{Gal}$ staining indicating Fgf $20^{\beta \mathrm{Gal}}$ expression in K14-Cre; $\beta$-Cat ${ }^{\Delta E \times 3}\left(\beta C a t^{a c t}\right) ; F g f 2 O^{\beta G a l /+}$ and K14$\mathrm{Cre} ; \beta-\mathrm{Cat}^{F / F}\left(\beta C a t^{C K O}\right) ; \mathrm{Fgf20} \mathrm{O}^{\beta G a l /+}$ embryos. by in situ hybridization and $\beta \mathrm{Gal}$ staining (Fig. 1B; Supplemental Fig. S1B,G). Fgf20 ${ }^{\beta \mathrm{Gal}}$ expression was detected throughout embryonic hair follicle morphogenesis in all pelage hair types (Supplemental Fig. S1A-F). To examine whether Fgf2O was regulated in vivo by Eda/Edar, we bred gain-of-function and loss-of-function alleles of Eda onto the $F g f 20^{\beta G a l /+}$ background and stained for $\beta \mathrm{Gal}$ activity. Activation of Eda in epidermis (K14-Eda and K14-Edar) resulted in increased $\mathrm{Fgf} 2 \mathrm{O}^{\beta \mathrm{Gal}}$ activity, while loss of Eda/Edar signaling $\left(E d a^{-1}\right)$ resulted in decreased Fgf20 ${ }^{\beta G a l}$ activity (Fig. 1B; Supplemental Fig. S2A,B).

Fgf20 was previously implicated as a downstream target of $\beta$-Catenin (Chamorro et al. 2005), and, as anticipated, transfection of a $\beta$-Catenin-Lef1 fusion protein increased the expression of a murine Fgf20 promoterluciferase reporter by 65 -fold (Fig. 1C). Consistent with these in vitro studies, in vivo activation (expression of the $\beta$-Cat ${ }^{\Delta E x 3}$ allele) or inhibition (conditional inactivation of $\beta$-Catenin using K14-cre) of epithelial Wnt/ $\beta$-Catenin signaling resulted in gain and loss of $\mathrm{Fgf} 20^{\beta \mathrm{Gal}}$ expression, respectively (Fig. 1D). These data indicate that epithelial Eda/Edar and Wnt/ $\beta$-Catenin signals activate Fgf20 expression in vivo.

\section{Fgf20 is required for guard hair formation}

During embryogenesis, different pelage hair types are induced in successive waves. Guard (tylotrich) hair follicles are the first to form, followed by formation of awl, auchene, and zigzag follicles (Schmidt-Ullrich and Paus 2005). To determine whether Fgf20 is functionally important for hair follicle formation, we examined hair from back skin of adult $F g f 20^{\beta G a l / \beta G a l}$ mice. Of the four morphologically distinct hair types, guard hairs were missing in $\mathrm{Fgf20^{ \beta Gal } / \beta \mathrm { Gal }}$ mice (Fig. 2A), while, awl, auchene, and zigzag hairs were readily identified and showed normal shaft morphology (Fig. 2B). To determine whether lack of Fgf20 affected the development of secondary and tertiary hair types, numbers of hairs from 3-wk-old Fgf $20^{\beta G a l /+}$ and $F g f 20^{\beta G a l / \beta G a l}$ mice were quantified. In heterozygous Fgf20 $0^{\beta \mathrm{Gal} /+}$ mice, guard hairs represented $2.2 \% \pm 1.1 \%$ of the total population. In $\mathrm{Fgf} 2 \mathrm{O}^{\beta \mathrm{Gal} / \beta \mathrm{Gal}}$ mice, guard hairs were not detected (Fig. 2C). Interestingly, the percentage of awl and auchene hairs was significantly reduced from $9.2 \% \pm 2.2 \%$ and $9.5 \% \pm 3.1 \%$, respectively, in $\mathrm{Fgf2} 2 \mathrm{O}^{\beta \mathrm{Gal} /+}$ mice to $1.8 \% \pm 1.9 \%$ and $2.8 \% \pm 1.7 \%$, respectively, in Fgf20 ${ }^{\beta G a l} / \beta$ Gal mice $(P<0.002$ and $P<$ $0.009, n=4$, respectively) (Fig. 2C). The percentage of zigzag hairs was increased from $79 \% \pm 3.5 \%$ in $\mathrm{Fgf20} \mathrm{O}^{\beta \mathrm{Gal} /+}$ mice to $95.4 \% \pm 3.5 \%$ in $F g f 20^{\beta G a l / \beta G a l}$ mice $(P<0.003)$ (Fig. 2C). Thus, Fgf2O is required for the formation of primary and most of the secondary hairs in mice.

\section{Fgf20 is required for the primary dermal condensation}

Prior to primary hair placode formation (E13.5), the skin of $F g f 20^{\beta G a l / \beta G a l}$ embryos was histologically indistinguishable from Fgf20 ${ }^{\beta G a l /+}$ embryos (data not shown). At E14.5, scanning electron microscope analysis suggested the absence of primary hair follicle primordia in $\mathrm{Fgf20^{ \beta Gal } / \beta \mathrm { Gal }}$ embryos (Fig. 2D), yet epithelial thickenings (placodes) 
Huh et al.

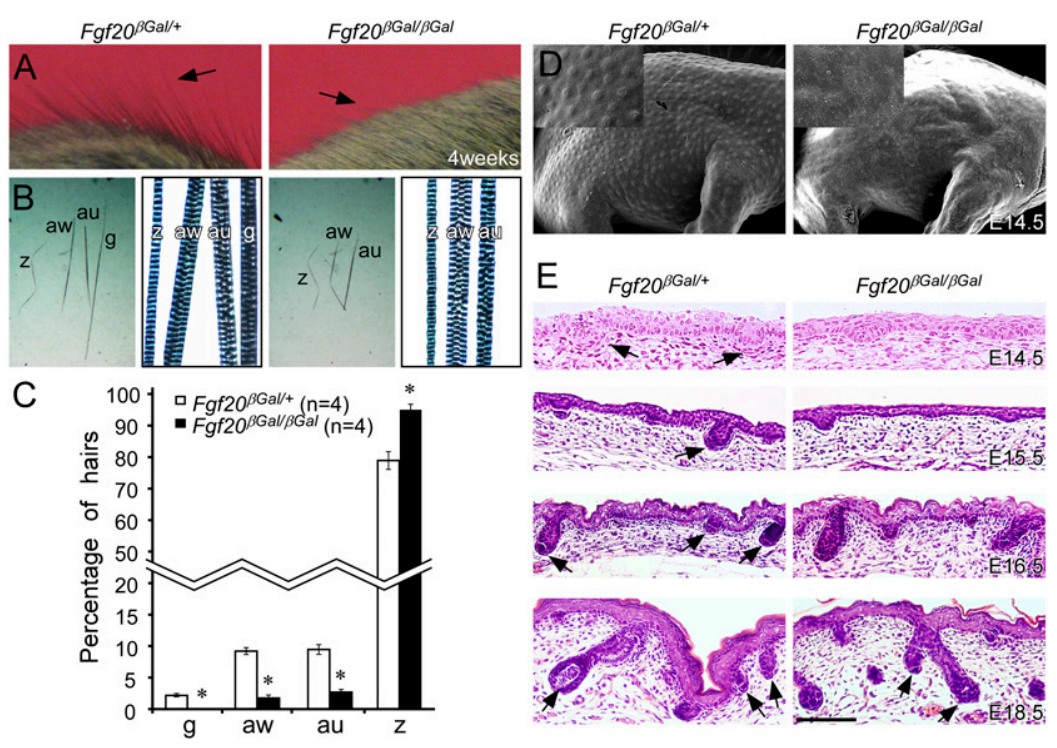

Figure 2. Loss of Fgf2O results in guard hair agenesis. (A) Image of 4-wk-old mice showing guard hair shafts sprouting from back skin in a $\mathrm{Fgf2O} \mathrm{O}^{\beta \mathrm{Gal} /+}$ mouse (arrow, left) but not in a Fgf20 $20^{\beta G a l / \beta G a l}$ mouse (right). (B) Image of hairs from back skin of a Fgf20 $0^{\beta G a l /+}(1$ eft) or Fgf20 $0^{\beta G a l / \beta G a l}$ (right) mouse. (C) Quantification of hair types from 3-wk-old mice. $F g f 20^{\beta G a l / \beta G a l}$ mice show complete loss of guard hairs and decreased awl and auchene hairs. (D) Scanning electron micrograph showing primary hair follicle primordia as round protrusions in a $F g f 20^{\beta G a l /+}$ embryo, but the surface of a $F g f 20^{\beta G a l / \beta G a l}$ embryo appears flat. (E) Histology of E14.5, E15.5, E16.5, and E18.5 skin from $F g f 2 O^{\beta G a l /+}$ and $F g f 2 O^{\beta G a l / \beta G a l}$ embryos. Arrows indicate dermal condensations. (Bottom right panel) Note the bifurcated hair follicle. (au) Auchene; (g) guard; (aw) awl; (z) zigzag. Bar, $100 \mu \mathrm{m}$.

were histologically evident in both $\mathrm{Fgf2O}^{\beta \mathrm{Gal} /+}$ and Fgf20 $0^{\beta G a l / \beta G a l}$ embryos (Fig. 2E). Strikingly, there was no histological evidence of dermal condensation formation in Fgf20 $0^{\beta G a l / \beta G a l}$ embryos (Fig. 2E). At E15.5, two types of hair follicles could be identified in $\mathrm{Fgf} 2 \mathrm{O}^{\beta \mathrm{Gal} / \beta \mathrm{Gal}}$ embryos-small and flat placodes (the majority) and follicles that had grown deeper into the dermis (occasional)but none of them were associated with dermal condensations (Fig. 2E). At E16.5 in Fgf20 ${ }^{\beta \mathrm{Gal} /+}$ embryos, the primary hair follicles reached the peg stage, and secondary hair placodes were formed. In contrast, in $\mathrm{Fgf20^{ \beta Gal } / \beta \mathrm { Gal }}$ embryos, most hair follicles were very small and were not associated with dermal condensations. However, sporadic primary hair placodes extended into the dermis to form a hair peg and were associated with a very small mesenchymal condensation (Fig. 2E). At E18.5 in Fgf20 ${ }^{\beta G a l / \beta G a l}$ embryos, some primary hair follicles were observed and were occasionally bifurcated, indicating an additional defect in hair follicle development (Fig. 2E). Tertiary placodes formed in both genotypes (Fig. 2E). These data show that Fgf2O is necessary for the formation of dermal condensations in primary hair follicles.

To examine Fgf20 signaling in the dermis and look for molecular evidence of dermal condensation, phospho-
Erk1/2, Sox2, Bmp4, p21, Inhba (Inhibin $\beta A$, previously known as activin $\beta A$ ), and $D k k 1$ expression was examined (Andl et al. 2002; Laurikkala et al. 2002; Schmidt-Ullrich and Paus 2005). At E14.5, phospho-Erk1/2 was detected in dermal cells as well as the epidermis of Fgf20 $\mathrm{O}^{\beta \mathrm{Gal} /+}$ embryos (Fig. 3A, left). In the absence of Fgf20, dermal phospho-Erk1/2 was not detected (Fig. 3A, right). Sox 2 is one of the earliest genes expressed in dermal condensations of primary and secondary, but not tertiary (zigzag), follicles (Driskell et al. 2009). By E13.75, P-Cadherinand $W n t 10 b$-positive epidermal foci could be detected in both Fgf2O $\mathrm{O}^{\beta G a l /+}$ and Fgf2O $\mathrm{O}^{\beta G a l / \beta G a l}$ embryos (Supplemental Fig. S3A,B), and, occasionally, P-Cadherin ${ }^{+}$foci were associated with Sox $2^{+}$cells in Fgf $20^{\beta G a l /+}$ embryos but not in $F g f 20^{\beta G a l / \beta G a l}$ embryos (Supplemental Fig. S3A). At E14.0 and E14.5, Sox2 expression was readily observed in dermal condensations in $\mathrm{Fg} 2 \mathrm{O}^{\beta \mathrm{Gal} /+}$ embryos. However, no Sox 2 expression was detected in $\mathrm{Fgf20} \mathrm{O}^{\beta \mathrm{Gal} / \beta \mathrm{Gal}}$ littermates at these stages (Fig. 3B,C). At E15.5, hair follicles were still devoid of Sox2 expression, in sharp contrast to control embryos (Fig. 3D). Consistent with the morphological absence of dermal condensations, focal expression of Bmp4, p21, Dkk1, and Inhba was also not detected in Fgf20 ${ }^{\beta \text { Gall } \beta \text { Gal }}$ dermis at E13.75-E14.5 (Fig. 3F-I; Supple-
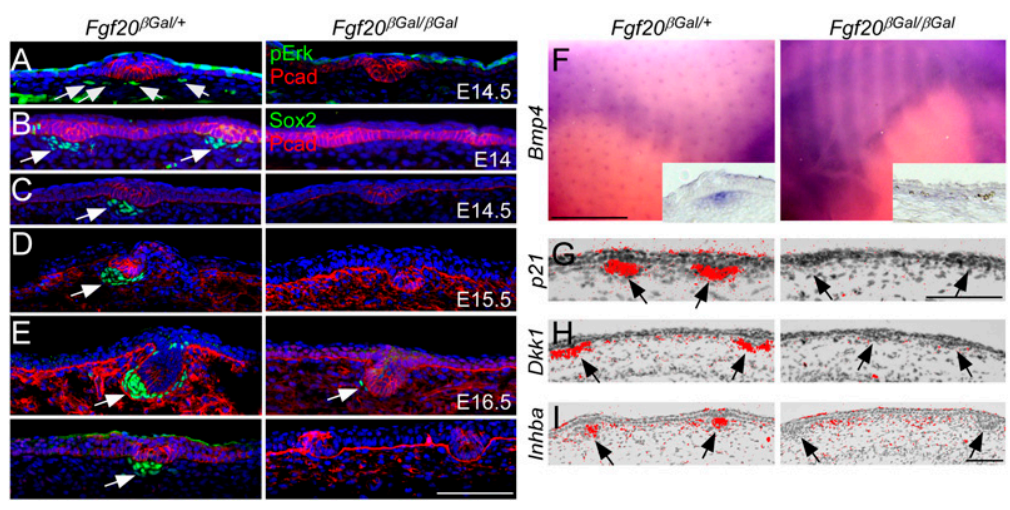

Figure 3. Fgf20 is required for dermal condensation development. (A) Phospho-Erk1/2 staining showing loss of Erk1/2 activity in dermis from $\mathrm{Fgf20} \mathrm{O}^{\beta \mathrm{Gal} / \beta \mathrm{Gal}}$ skin (left) compared with control (right). (B-E) Coimmunostaining for Sox2 and P-Cadherin (Pcad) showing Sox $2^{+}$dermal condensations at E14 $(B)$, E14.5 $(C)$, E15.5 $(D)$, and E16.5 $(E)$ in Fgf20 ${ }^{\beta G a l /+}($ left) but not in $F g f 20^{\beta G a l / \beta G a l}$ (right) embryo skin. $(F-I)$ In situ hybridization of Bmp4 $(F), p 21(G), D k k 1(H)$, and Inhba (I) showing normal dermal expression in Fgf20 $0^{\beta G a l /+}($ left $)$ embryos and loss of expression in Fgf20 ${ }^{\beta G a l / \beta G a l}$ embryos (right). Bar, $100 \mu \mathrm{m}$. 
mental Fig. S3C,D). Quantitation of $B m p 4^{+}$foci showed an absence of primary dermal condensations in $F g f 20^{\beta G a l / \beta G a l}$ embryos (Supplemental Fig. S3E). We conclude that in the absence of $F g f 20$, primary hair placodes were devoid of any known morphological and molecular evidence of dermal condensation formation.

Next, we examined dermal condensation formation and maturation at later stages of development. At E16.5, Sox 2 and $B m p 4$ were expressed in primary and secondary follicles of control embryos, while $\mathrm{Fgf20} \mathrm{O}^{\beta \mathrm{Gal} / \beta \mathrm{Gal}}$ embryos showed rare Sox $2^{+}$cells in the few follicles that had advanced further (Fig. 3E; Supplemental Fig. S4A,B).

However, the majority of $\mathrm{Fg} f 20^{\beta \mathrm{Gal} / \beta \mathrm{Gal}}$ hair follicles remained at the placode/germ stage and were negative for all condensation markers analyzed (Fig. 3E; Supplemental Fig. S4A). Corin, a marker of maturing dermal papillae of all hair types (Enshell-Seijffers et al. 2008), was also absent from $F g f 20^{\beta G a l / \beta G a l}$ embryos but was readily detected in primary follicles of control embryos at E16.5 (Supplemental Fig. S4B). At E17.5, Sox2 and Corin were expressed in both primary and secondary follicles of $\mathrm{Fgf2 \textrm {O } ^ { \beta G a l + }}$ embryos, while in $\mathrm{Fgf20^{ \beta Gal/ \beta Gal }}$ skin, many (secondary) follicles showed an absence or low level of expression of both markers (Supplemental Fig. S4C). CD133, another marker of dermal condensations (Ito et al. 2007), was expressed at E15.5 and E16.5 (Supplemental Fig. S4D,E, left) in Fgf2 $\mathrm{O}^{\beta G a l /+}$ embryos. However, CD133 expression was not detected in Fgf20 3 Gal/BGal embryos at the same time point (Supplemental Fig. S4D,E, right). Together, these data indicate that Fgf20 is critical for formation of primary dermal condensations and that dermal condensations of secondary follicles were also affected in $F g f 20^{\beta G a l / \beta G a l}$ embryos, but to a lesser extent.
Fgf20 modulates Eda/Edar and Wnt/ $\beta$-Catenin

but not the Sonic hedgehog (Shh) signaling cascade

To assess the consequences of loss of Fgf2O and the absence of dermal condensation formation on hair placode development, we analyzed the expression of several key epithelial genes in $F g f 20^{\beta G a l / \beta G a l}$ embryos at E14.0-E14.5 (Schmidt-Ullrich et al. 2006; Pummila et al. 2007). Unexpectedly, Wnt10b, a well-characterized hair placode marker, as well as Lef1 and $\beta$-Catenin showed a stripelike expression pattern in the epidermis in $\mathrm{Fgf2} \mathrm{O}^{\beta \mathrm{Gal} / \beta \mathrm{Gal}}$ embryos (Fig. 4A-C). Also, Fgf20 ${ }^{\beta \mathrm{Gal}}$ activity showed a similar pattern in $F g f 20^{\beta G a l / \beta G a l}$ embryos, in contrast to the punctuate expression seen in $\mathrm{Fgf2} \mathrm{O}^{\beta \mathrm{Gal} /+}$ embryos (Fig. 4D). During hair follicle induction, Wnt/ $\beta$-Catenin signaling is active in both the epidermis and dermis (DasGupta and Fuchs 1999; Zhang et al. 2008, 2009). In control embryos, Axin2, a target of canonical Wnt signaling, was expressed in the epidermis in primary hair follicles and in the underlying dermis. However, in Fgf20 ${ }^{\beta G a l / \beta G a l}$ embryos, dermal expression of Axin2 was severely decreased, while epidermal expression was slightly broader and more intense (Fig. 4E). Immunohistochemical detection of $\beta$-Catenin showed nuclear localization (Fig. 4F, arrows) in the dermal condensations of control embryos but reduced nuclear localization in Fgf20 $0^{\beta G a l / \beta G a l}$ embryos (Fig. 4F). Furthermore, nuclear Lef1 protein was reduced in the dermis of Fgf2O $0^{\beta G a l / \beta G a l}$ embryos compared with littermate controls (Fig. 4G). These data indicate that Fgf2O inhibits canonical Wnt/ $\beta$ Catenin signaling in the epidermis but activates it in dermal condensations.

Patterning of hair follicles is thought to be the result of a reaction-diffusion mechanism involving the interaction of diffusible substances that are commonly referred

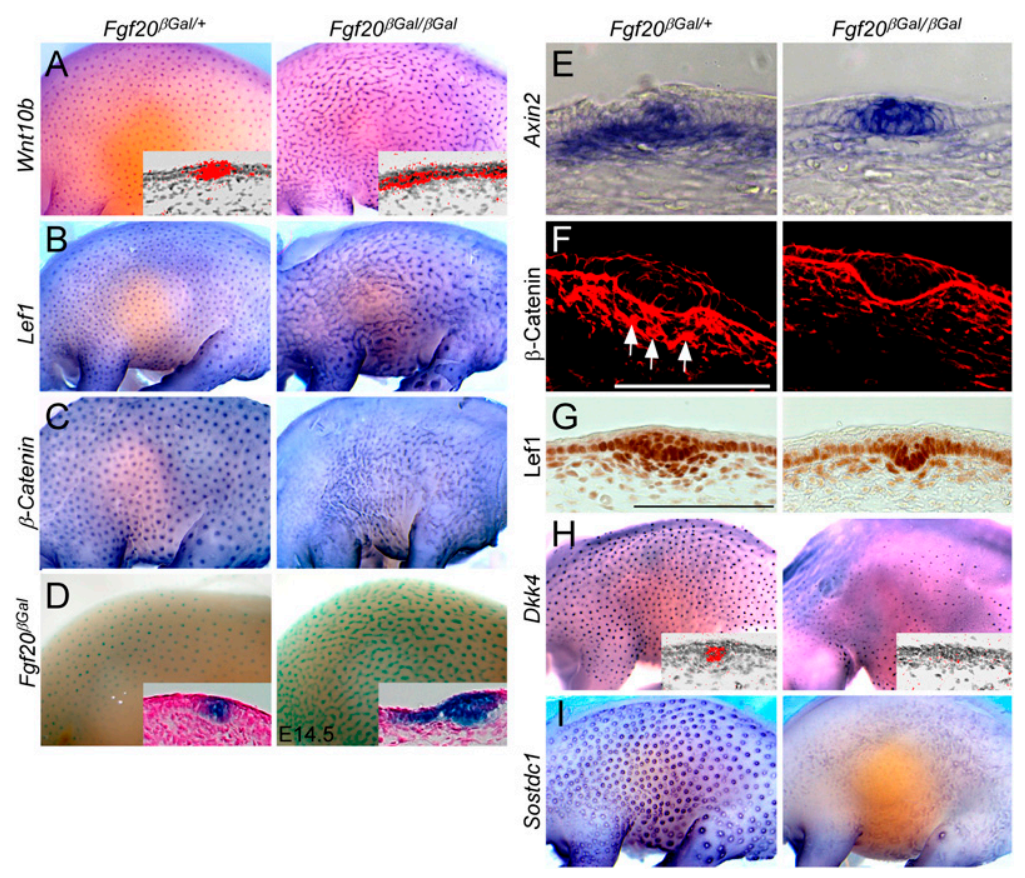

Figure 4. Fgf20 modulates epithelial and mesenchymal Wnt/ $\beta$-Catenin signaling during primary hair placode formation. $(A-C)$ In situ hybridization for Wnt10b $(A)$, Lef1 $(B)$, and $\beta$-Catenin $(C)$ showing stripe-like expression in $F g f 2 O^{\beta G a l / \beta G a l}$ embryos compared with Fgf20 $0^{\beta G a l /+}$ embryos. (D) Fgf $20^{\beta G a l}$ staining showing increased interfollicular $\beta \mathrm{Gal}$ staining in $F g f 20^{\beta G a l / \beta G a l}$ embryos compared with Fgf2O $2 O^{\beta G a l /+}$ embryos. (E) In situ hybridization of Axin2 showing increased expression in placodal cells but decreased expression in dermal cells in Fgf20 ${ }^{\beta G a l / \beta G a l}$ embryos compared with $F g f 20^{\beta G a l /+}$ embryos. $(F)$ Immunostaining for $\beta$-Catenin showing loss of $\beta$-Catenin nuclear localization (arrows) in dermal cells in Fgf $20^{\beta G a l / \beta G a l}$ embryos compared with Fgf2O $20^{\beta G a l /+}$ embryos. (G) Immunostaining for Lef1 shows strong nuclear expression in the dermal condensation of $\mathrm{Fgf} 2 \mathrm{O}^{\beta \mathrm{Gal} /+}$ but not in $\mathrm{Fgf} 2 \mathrm{O}^{\beta \mathrm{Gal} / \mathrm{\beta Gal}}$ embryos at E14.5. $(H, I)$ In situ hybridization for Dkk4 $(H)$ and Sostdc1 $(I)$ showing severely reduced expression in $F g f 2 O^{\beta G a l / \beta G a l}$ embryos compared with Fgf20 ${ }^{\beta G a l /+}$ embryos. Bar, $100 \mu \mathrm{m}$. 
Huh et al.

to as "the activator" and "the inhibitor" (Kondo and Miura 2010). Activating Wnt ligands and their soluble inhibitors (Dkk1 and Dkk4) have been proposed to function at the core of this patterning process (Andl et al. 2002; Sick et al. 2006). The "spreading" of Wnt10b expression in Fgf $20^{\beta G a l / \beta G a l}$ embryos is consistent with predictions of reaction-diffusion models in situations where activator concentrations become saturating (Mou et al. 2006; Kondo and Miura 2010). Absence of Dkk1 (Fig. 3G) together with the severe down-regulation of Dkk4 (Fig. 4H) and Sostdc1 (Ectodin and Wise) (Fig. 4I), another Wnt antagonist regulating hair placode size (Närhi et al. 2012) in Fgf20 ${ }^{\beta G a l / \beta G a l}$ embryos, may explain the observed phenomenon.

We next analyzed expression of Edar and observed severely decreased levels in Fgf20 $0^{\beta G a l / \beta G a l}$ embryos compared with $F g f 20^{\beta G a l /+}$ embryos (Fig. 5A). I $\kappa-B \alpha$, the downstream target of Eda/Edar (Schmidt-Ullrich et al. 2006; Pummila et al. 2007), was similarly decreased in Fgf20 ${ }^{\beta G a l / \beta G a l}$ embryos (Fig. 5B). Thus, loss of Fgf2O suppresses Edar expression, leading to reduced Edar signaling. Reduced expression of Edar was surprising given that placodal Wnts have been shown to up-regulate Edar expression (Zhang et al. 2009). These data suggest that in addition to regulation by Wnts, Edar expression also depends on signals emanating from the dermal condensation.

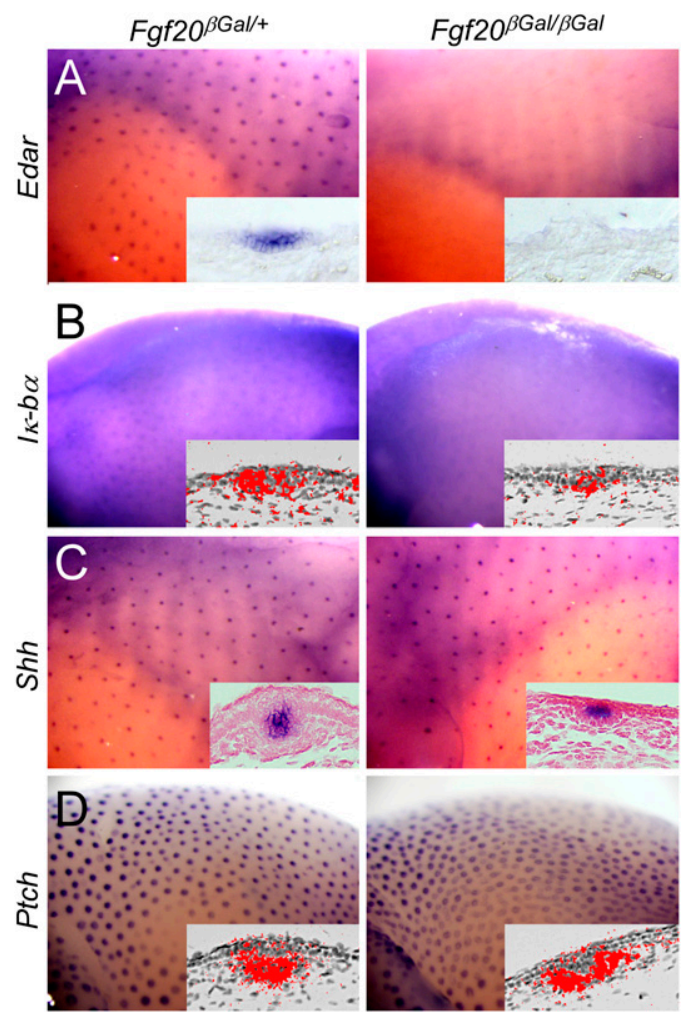

Figure 5. Fgf2O modulates Eda/Edar but not Shh signaling during primary hair placode formation. $(A-B)$ In situ hybridization for Edar $(A)$ and $I \kappa-B \alpha(B)$ showing decreased expression in Fgf20 $0^{\beta G a l / \beta G a l}$ embryos compared with $\mathrm{Fgf2O}^{\beta \mathrm{Gal} /+}$ embryos. $(C, D)$ In situ hybridization of Shh $(C)$ and Patched1 (Ptch) (D) showing unaltered expression in Fgf20 $0^{\beta G a l / \beta G a l}$ embryos compared with $\mathrm{Fgf20^{ \beta Gal/+ }}$ embryos. Bar, $100 \mu \mathrm{m}$.
Shh is expressed in hair placodes and functions to induce proliferation of hair placode epithelial cells, enabling growth into the dermis and the formation of the hair peg (St-Jacques et al. 1998; Chiang et al. 1999). Expression levels and the pattern of Shh and its target gene, Patched1, were comparable in control and Fgf20 ${ }^{\beta G a l / \beta G a l}$ embryos at E14.5 (Fig. 5C,D; Supplemental Fig. S5A,B). These data show that Shh signaling functions independently of Fgf2O and that, despite intact Shh signaling, down-growth of primary hair follicles was impaired in Fgf20 ${ }^{\beta G a l / \beta G a l}$ embryos.

We also noted that in Fgf20 $0^{\beta G a l / \beta G a l}$ embryos, Shh expression was confined to placodal cells, unlike Wnt10b and $\beta \mathrm{Gal}\left(\mathrm{Fgf} 20^{\beta \mathrm{Gal}}\right)$, whose expression expanded into the interfollicular epidermis (cf. Figs.4A,D and 5C). Expression of Shh (and primary placode formation) is thought to require both Wnt/ $\beta$-Catenin (Andl et al. 2002; Fuchs 2007) and Eda/Edar (Schmidt-Ullrich et al. 2006; Pummila et al. 2007). In Fgf20 $0^{\beta G a l / \beta G a l}$ embryos, Eda/Edar signaling is greatly reduced (Fig. 5A,B). To address whether Shh expression and placode formation in $\mathrm{Fgf} 20^{\beta \mathrm{Gal} / \beta \mathrm{Gal}}$ embryos requires Eda, we generated mice deficient in both Fgf2O and Eda. Eda ${ }^{-/-} ; F_{g f 2 O^{\beta G a l /+}}$ and Eda ${ }^{-/-}$; Fgf $20^{\beta G a l /} \beta$ Gal embryos lacked all expression of $S h h$ at E14.5 (Supplemental Fig. S5C,D). These data indicate that Edar signaling, although strongly reduced, is nevertheless necessary for Shh expression in Fgf2 $0^{\beta G a l / \beta G a l}$ embryos. We propose that the relatively normal patterning of Shh expression and morphological placodes in Fgf20 mutants is achieved via simultaneous activation of Edar and Wnt/ $\beta$-Catenin pathways.

\section{Fgf20 functions downstream from Eda/Edar and $W n t / \beta$-Catenin signaling to form dermal condensations}

If Fgf2O is the critical signal for dermal condensation formation downstream from Wnt/ $\beta$-Catenin and Eda/Edar signaling, then loss of Fgf20 would block formation of primary dermal condensations in Eda or Wnt/ $\beta$-Catenin gain-of-function (K14-Eda or K14-Cre; $\beta$-Cat $\left.{ }^{\Delta E \times 3}\right)$ embryos. We addressed the signaling hierarchy between Fgf20 and Eda by comparing $\mathrm{Fgf2} 2 \mathrm{O}^{\beta \mathrm{Gal} /+}$ and $\mathrm{Fgf2} \mathrm{O}^{\beta \mathrm{Gal} / \beta \mathrm{Gal}} \mathrm{em}$ bryos with K14-Eda;Fgf20 ${ }^{\beta G a l /+}$ and K14-Eda;Fgf20 ${ }^{\beta G a l} / \beta \mathrm{Gal}$ embryos. Overexpression of $E d a$ resulted in enlarged hair placodes associated with larger Sox $2^{+}$dermal condensations (Fig. 6A; Supplemental Fig. S6), consistent with previously reported data (Mustonen et al. 2004). Interestingly, the size of the primary hair placodes in K14-Eda embryos (indicated by P-Cadherin staining) was unaffected by loss of Fgf20, yet dermal condensations were absent (Fig. 6A; data not shown). The inability of excess Eda to rescue dermal condensation formation in K14-Eda; $F g f 20^{\beta G a l / \beta G a l}$ embryos could be due to reduced Edar expression due to the absence of Fgf20 (Fig. 5A). However, we found robust induction of $I \kappa-B \alpha$ expression by K14-Eda in both heterozygous and $F g f 20^{\beta G a l / \beta G a l}$ embryos (Fig. 6B), excluding this possibility. These data support a model in which Fgf20 is required for dermal condensation formation downstream from Eda/Edar signaling. 


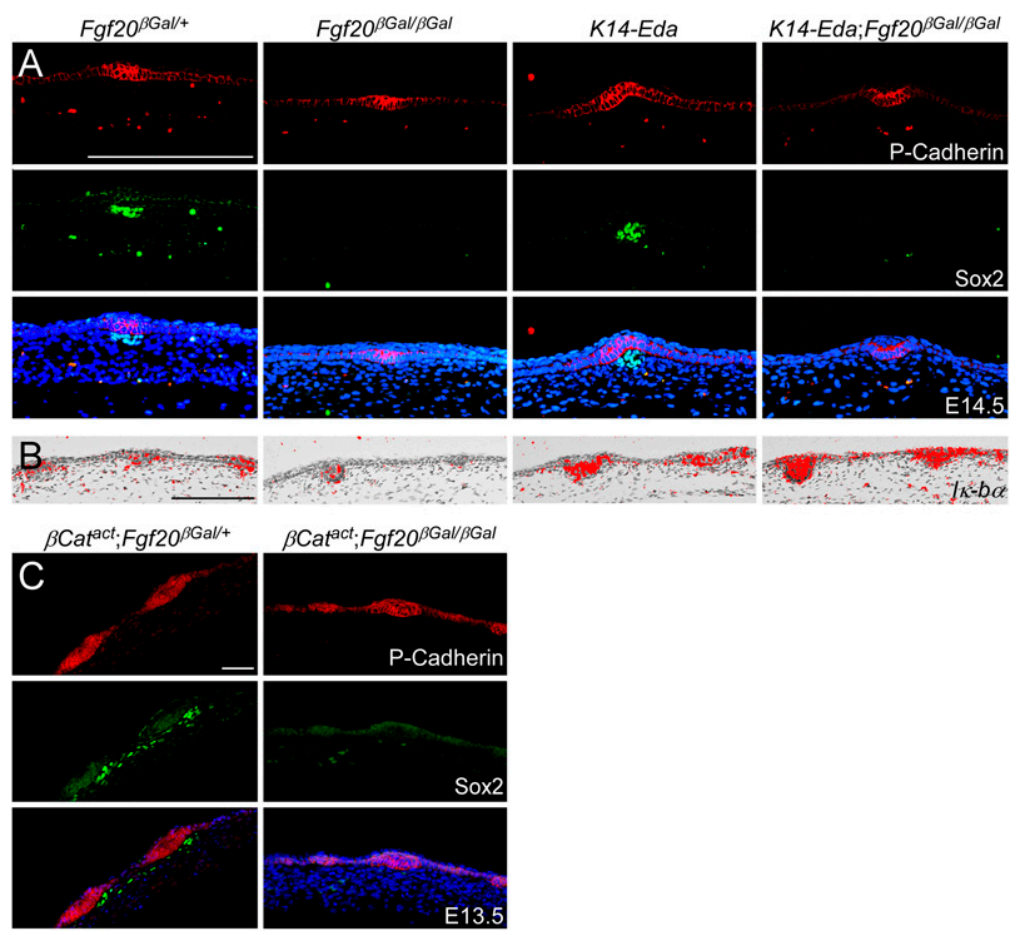

Figure 6. Eda/Edar and Wnt $/ \beta$-Catenin signalinginduced dermal condensation is Fgf20-dependent. (A) Coimmunostaining for Sox2 and P-Cadherin showing that Sox $2^{+}$dermal condensation induced by Eda overexpression (K14-Eda) has been abolished in $\mathrm{Fg} f 20^{\beta G a l / \beta G a l}$ embryos compared with $\mathrm{Fgf} 2 \mathrm{O}^{\beta \mathrm{Gal} /+}$ embryos at E14.5. (B) In situ hybridization for $I \kappa-B \alpha$ showing increased expression in K14-Eda and K14$E d a ; F g f 20^{\beta G a l / \beta G a l}$ embryos at E14.5. (C) Coimmunostaining for Sox2 and P-Cadherin at E13.5 showing that Sox $2^{+}$dermal condensations induced by K14$\mathrm{Cre} ; \beta-\mathrm{Cat}^{\Delta E \times 3}\left(\beta \mathrm{Cat}^{a c t}\right)$ were absent in Fgf20 ${ }^{\beta \mathrm{Gal} /}$ $\beta G a l$ embryos compared with $F g f 20^{\beta G a l /+}$ embryos, yet premature induction of placodes was unaffected by loss of Fgf20 (cf. Supplemental Fig. S1A). Bar, $100 \mu \mathrm{m}$.

To determine whether epidermal Wnt/ $\beta$-Catenin signaling could rescue dermal condensation formation in the absence of Fgf20, we compared Fgf2 $0^{\beta G a l /+}$ and

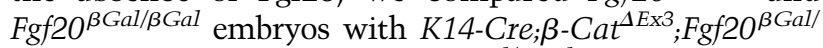
${ }^{+}$and $\mathrm{K14}-\mathrm{Cre} ; \beta-\mathrm{Cat}^{\Delta E \times 3} ; \mathrm{Fgf20}{ }^{\beta G a l / \beta G a l}$ embryos. Forced activation of epidermal Wnt/ $\beta$-Catenin signaling in Fgf $20^{\beta \mathrm{Gal} /+}$ embryos resulted in ectopic dermal condensation formation, as shown by expanded Sox2 expression at E13.5 (cf. Figs. 3B, 6A and 6C). However, in K14-Cre; $\beta$-Cat ${ }^{\Delta E x 3} ; F g f 20^{\beta G a l / \beta G a l}$ embryos, dermal condensations failed to form, and Sox2 expression was not detected (Fig. 6C, right). Despite the absence of dermal condensations in K14-Cre; $\beta$-Cat ${ }^{\Delta E x 3}$ embryos lacking Fgf20, hair placodes were precociously induced, as evidenced by P-Cadherin expression (cf. Fig. 6C and Supplemental Fig. S1A). Together, these data indicate that Fgf20 is not required for placode formation but functions downstream from Eda/Edar and Wnt/ $\beta$-Catenin signaling to induce formation of the dermal condensations, without which guard hairs cannot form.

\section{Discussion}

Hair follicle induction is thought to proceed via an ordered series of epithelial-mesenchymal interactions (Hardy 1992). An initial mesenchymal signal instructs the epithelium to make a placode; a second, epithelial signal is believed to pass from the placode to mesenchyme to induce formation of a dermal condensation, and a second dermal signal drives proliferation and downgrowth of placodal cells. A major obstacle in deciphering the details and exact order of the inductive events during hair follicle formation has been the lack of knowledge on the identity of these key signals. Histological studies on nascent whisker primordia indicated the presence of dermal condensations prior to epithelial thickenings (Van Exan and Hardy 1980). In contrast, some placode markers show a labile prepattern in $E d a^{-1-}$ embryos where no primary dermal condensations have been recognized (Laurikkala et al. 2002; Mou et al. 2006; Fliniaux et al. 2008; Zhang et al. 2009|, suggesting an "epitheliumfirst" patterning process. On the other hand, a recent study showed that Wnt/ $\beta$-Catenin activity (Axin $2^{\beta \mathrm{Gal}}$ ) becomes patterned in both the epithelium and dermis simultaneously before the appearance of morphologically distinct placodes or condensations (Zhang et al. 2009). Thus, it is controversial as to whether patterning first arises in the epithelium or the mesenchyme.

Here, we show that in the absence of Fgf20, a regular array of primary hair placodes forms in the absence of discernible dermal condensations. Although we cannot rule out the existence of an unknown asymmetric signal in Fgf20 mutant dermis, based on the morphological evidence and the absence of a wealth of dermal condensation markers (Sox2, p21, Dkk1, Inhba, $\beta$-Catenin, Axin2, $B m p 4$, Corin, CD133), we find the presence of such a cue unlikely. Rather, we interpret our data to indicate that the embryonic epithelium-once committed to form hairhas the ability to generate periodicity and that patterning originates initially in the epithelium and can arise independent of dermal condensations. We propose that the primary inductive cue is uniform rather than patterned, a conclusion supported by functional experiments showing that periodic dermal $\mathrm{Wnt} / \beta$-Catenin activity depends on epithelial $\beta$-Catenin (Zhang et al. 2009). Our conclusions appear seemingly contradictory to studies using 
mesenchymal-epithelial tissue chimeras, which indicate that hair patterning is under the control of the dermis (Kollar 1970; Dhouailly 1973). However, these tissue recombination experiments can be explained by a mesenchymal-derived inductive cue, even if uniformly expressed, influencing the spacing of hair placodes; for example, by altering reaction-diffusion dynamics.

Until now, molecular mechanisms governing dermal condensation formation have been poorly defined (Sennett and Rendl 2012). Previous studies have implicated Shh and platelet-derived growth factor in dermal morphogenesis, but in mice lacking either factor, dermal papillae form but are reduced in size (St-Jacques et al. 1998; Chiang et al. 1999; Karlsson et al. 1999). This indicates a role in dermal papilla maturation/maintenance, a function that is thought to require the Bmp inhibitor Noggin (Woo et al. 2012). Additionally, Wnts have been proposed to participate in the generation of dermal condensations (Zhang et al. 2009; Sennett and Rendl 2012). However, forced activation of dermal $\beta$-Catenin is not sufficient to induce dermal cells to adopt a dermal condensation fate (Chen et al. 2012), while activation of epithelial $\beta$-Catenin is necessary for the formation of dermal condensations (Zhang et al. 2009; this study), implying involvement of a Wnt-dependent epithelial signal for the formation of dermal condensations. Here, we identify Fgf20, downstream from Eda/NF-кB and Wnt/ $\beta$-Catenin, as an essential signal required for dermal condensation formation in primary and most secondary hair follicles. The absence of an effect on tertiary hair follicles suggests either redundancy with another Fgf ligand or that another, as yet unidentified, signal is required for tertiary hair follicle formation. The Fgf receptor mediating the Fgf20 signal is also not defined; however, Fgfrl is a good candidate, as it is expressed at high levels in the upper dermis at the time of hair follicle induction (Chen et al. 2012). Additionally, Fgfr2 is expressed at low levels in the developing dermis (Richardson et al. 2009) and therefore could have redundant function with Fgfr1. Further support for a key evolutionarily conserved role for Fgf20 signaling in epidermal appendage formation comes from the scaleless mutant chicken, which lacks almost all feathers and scales due to a nonsense mutation in Fgf20 (Wells et al. 2012). In addition, in vitro studies have implicated Fgfs in dermal condensation formation in chicken dermis (Song et al. 2004; Lin et al. 2009).

It is well established that dermal condensation/papilla cells can induce hair follicle development even when combined with non-hair-forming epithelium, and thus the underlying mechanism is a focus of regenerative medicine research (Hardy 1992; Yang and Cotsarelis 2010). However, therapeutic efforts to use dermal papilla cells have been hampered by their low abundance, difficulty in isolation, and loss of hair follicle-inducing properties in culture (Yang and Cotsarelis 2010). These studies, which identify Fgf20 as a signal essential for dermal condensation formation, combined with the knowledge of how dermal cells acquire hair follicle-inducing capacity, will be useful in future attempts to treat hair loss.

\section{Materials and methods}

\begin{abstract}
Mice
Fgf2O ${ }^{\beta G a l /+}, E d a^{-/+}$, K14-Eda, K14-Edar, $\beta$-Cat ${ }^{F / F}, \beta$-Cat ${ }^{\Delta \mathrm{Ex} 3}$, K14-cre,

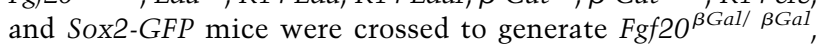
$E_{\text {Eda }}^{-/-}$, K14-Eda;Fgf20 ${ }^{\beta G a l /} \beta$ GGal, K14-Edar;Fgf20 ${ }^{\beta G a l / \beta G a l}$, K14-cre;

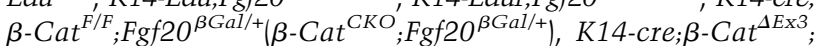

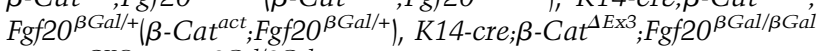
$\left(\beta\right.$-Cat $\left.{ }^{C K O} ; F^{\prime} f 2 O^{\beta G a l / \beta G a l}\right)$, and K14-Eda;Sox2-GFP mouse lines (Harada et al. 1999; Dassule et al. 2000; Brault et al. 2001; Laurikkala et al. 2002; D'Amour and Gage 2003; Mustonen et al. 2004; Huh et al. 2012). Mice were maintained on a mixed genetic background, and littermates were used as controls as indicated. All animal studies were carried out in accordance with the guidelines and approval from the Washington University Animal Studies Committee and Finnish National Board of Animal Experimentation.
\end{abstract}

\section{Electron microscopy}

Embryos were fixed in 4\% paraformaldehyde in PBS over two nights; dehydrated in a graded series of $50 \%, 70 \%, 94 \%$, and $100 \%$ ethanol for $30 \mathrm{~min}$ each; and subjected to critical point drying (Bal-Tech, CPD 030) and platinum coating (Quorum, Q150TS coater). Samples were viewed with FEI Quanta 250 scanning electron microscope.

\section{Luciferase assay}

The mouse Fgf20 promoter construct was generated by subcloning a 5.2 -kb fragment of the proximal promoter region containing putative $\beta$-Catenin/TCF-binding sites into the firefly luciferase reporter plasmid pGL3 Basic (Promega). For transfection, HEK293T cells were seeded at $3 \times 10^{5}$ cells per well in gelatincoated six-well plates. The following day, $950 \mathrm{ng}$ of luciferase reporter plasmid was cotransfected with $500 \mathrm{ng}$ of an expression vector coding for dominant active Lef1- $\beta$-Catenin fusion protein (Wong et al. 2002) or corresponding empty vectors and $50 \mathrm{ng}$ of Renilla luciferase vector pRL-TK (Promega) using Fugene 6 transfection reagent (Roche). After $24 \mathrm{~h}$, luciferase activities were measured as described (Fliniaux et al. 2008). Three independent experiments with triplicate samples were performed.

\section{Quantitative RT-PCR ( $q R T-P C R)$}

E14.5 $\mathrm{Eda}^{-1-}$ back skins were dissected, cut into halves along the midline, and maintained for $2 \mathrm{~h}(n=12)$ or $4 \mathrm{~h}(n=6)$ in a $30-\mu \mathrm{L}$ hanging drop of DMEM, 10\% FCS, glutamine, and penicillinstreptomycin. For each treatment, one half was used as a control, while the other half was supplemented with $250 \mathrm{ng} / \mathrm{mL}$ recombinant Fc-Eda-A1 (Gaide and Schneider 2003). RNA isolation and qRT-PCR were done as previously described (Fliniaux et al. 2008). Transcript number was quantified by comparing the sample data against a dilution series of PCR products of the gene of interest. Expression of Fgf2O was normalized against Ranbp1.

\section{$\beta$ Gal staining}

Embryos at various stages of development were fixed overnight in Mirsky's fixative (National Diagnostics), washed three times in PBS, and incubated in $\beta$ Gal-staining solution $\left(2 \mathrm{mM} \mathrm{MgCl}_{2}\right.$, $35 \mathrm{mM}$ potassium ferrocyanide, $35 \mathrm{mM}$ potassium ferricyanide, $1 \mathrm{mg} / \mathrm{mg} \mathrm{X-Gal} \mathrm{in} \mathrm{PBS)} \mathrm{until} \mathrm{optimal} \mathrm{color} \mathrm{development} \mathrm{was}$ observed. Samples were washed in PBS, fixed in $10 \%$ formalin, and imaged under a dissecting microscope. For staining histo- 
logical sections, samples were cryosectioned, washed with PBS, and incubated in $\beta \mathrm{Gal}$-staining solution. Embryos were photographed on an Olympus SZX12 stereo microscope.

\section{Histology}

Embryos or dissected tissues were fixed in $4 \%$ PFA overnight at $4^{\circ} \mathrm{C}$, dehydrated, embedded in paraffin, and serially sectioned. For plastic histology (E14.5), tissues were embedded in Historesin as specified by the manufacturer (Leica) and stained with haematoxylin and eosin.

\section{Immunohistochemistry}

Embryos were embedded in OCT, frozen, and cryosectioned at $12 \mu \mathrm{m}$. Alternatively, paraffin-embedded tissues were serially sectioned at $7 \mu \mathrm{m}$, dewaxed, rehydrated, and microwaved (650 W) for $10 \mathrm{~min}$ in $10 \mathrm{mM}$ sodium citrate buffer ( $\mathrm{pH}$ 6.0). Sections were washed with PBS and blocked with $0.1 \%$ Triton X-100 and $0.5 \%$ donkey serum. Primary antibodies were added to the section and incubated in a humidified chamber overnight at $4^{\circ} \mathrm{C}$. Sections were washed and incubated with secondary antibody for $1 \mathrm{~h}$ at room temperature. Samples were washed three times with PBS, placed on cover slips with Vectashield (Vector Laboratories), and photographed using a Zeiss LSM 700 confocal microscope or Zeiss Axio Imager M2. The primary antibodies used were as follows: $\beta \mathrm{Gal}$ (1:500; abcam), Sox2 (1:500 [Millipore] or 1:200 [Santa Cruz Biotechnology]), P-Cadherin (1:100 [gift from Dr. Takeichi] or 1:50 [R\&D Systems]), Corin (1:800; gift from Dr. Morgan), $\beta$-Catenin (1:200; R\&D Systems), Lef1 (1:1000; Cell Signaling Technology), phospho-Erk1/2 (1:200; Cell Signaling Technology), and CD133 (1:500; Millipore).

\section{In situ hybridization}

For whole-mount in situ hybridization, embryos were fixed in $4 \%$ PFA overnight at $4^{\circ} \mathrm{C}$, washed in PBS, and dehydrated in increasing concentrations of methanol. Embryos were rehydrated, washed with hybridization solution, and incubated overnight with digoxigenin-labeled RNA probes. After washing, embryos were incubated with anti-digoxigenin antibody conjugated with alkaline phosphatase (Roche), and the color reaction was performed using alkaline phosphate substrate (Roche). Alternatively, whole-mount in situ hybridization was performed with InSituPro robot (Intavis AG) as previously described (Fliniaux et al. 2008). Embryos were photographed on an Olympus SZX12 stereo microscope. The probes used were as follows: Bmp4, p21, Dkk1, Inhba, Bmp7, Edar, IкB $\alpha$, Wnt10b, Fgf20, Axin2, Shh, Patched, Lef1, $\beta$-Catenin, Dkk4, and Sostdc1 (Ectodin) (Andl et al. 2002; Laurikkala et al. 2002; Mustonen et al. 2004; Schmidt-Ullrich et al. 2006; Fliniaux et al. 2008; Närhi et al. 2008). Radioactive in situ hybridization was performed on paraffin sections according to standard protocols using probes labeled with ${ }^{35}$ S-UTP. Dark-field images were inverted, linearly thresholded, and combined with bright-field images in Adobe Photoshop CS4.

\section{Hair and dermal condensation counting}

Different types of hairs were counted from 3-wk-old mice. More than 200 hairs were counted from each genotype. Dermal condensations were counted using E14.5 whole-mount Bmp4 in situ staining samples.

\section{Statistics}

Nonparametric Mann-Whitney $U$-test was used for statistical analysis of luciferase assay data. qRT-PCR data were analyzed with the nonparametric Wilcoxon signed-rank test for paired samples. $P<0.05$ was considered to be significant.

\section{Acknowledgments}

We thank B. Morgan for providing the Corin antibody; C. Smith for mouse husbandry; I. Thesleff, J. Jernvall, and R. Kopan for critical reading of the manuscript; and M. Mäkinen, R. Santalahti, and R. Savolainen for technical assistance. This work was funded by the Department of Developmental Biology, contributions from Edward and Linda Ornitz; NIH grant HD049808; NIH support grants, P30DC04665, P30DK052574, and P30AR057235; the Academy of Finland; the Sigrid Jusélius Foundation; Helsinki Graduate Program in Biotechnology and Molecular Biology; and Viikki Doctoral Program in Molecular Biosciences.

\section{References}

Andl T, Reddy ST, Gaddapara T, Millar SE. 2002. WNT signals are required for the initiation of hair follicle development. Dev Cell 2: 643-653.

Brault V, Moore R, Kutsch S, Ishibashi M, Rowitch DH, McMahon AP, Sommer L, Boussadia O, Kemler R. 2001. Inactivation of the $\beta$-catenin gene by Wnt1-Cre-mediated deletion results in dramatic brain malformation and failure of craniofacial development. Development 128: 1253-1264.

Chamorro MN, Schwartz DR, Vonica A, Brivanlou AH, Cho KR, Varmus HE. 2005. FGF-20 and DKK1 are transcriptional targets of $\beta$-catenin and FGF-20 is implicated in cancer and development. EMBO J 24: 73-84.

Chen D, Jarrell A, Guo C, Lang R, Atit R. 2012. Dermal $\beta$-catenin activity in response to epidermal Wnt ligands is required for fibroblast proliferation and hair follicle initiation. Development 139: 1522-1533.

Chiang C, Swan RZ, Grachtchouk M, Bolinger M, Litingtung Y, Robertson EK, Cooper MK, Gaffield W, Westphal H, Beachy PA, et al. 1999. Essential role for Sonic hedgehog during hair follicle morphogenesis. Dev Biol 205: 1-9.

D'Amour KA, Gage FH. 2003. Genetic and functional differences between multipotent neural and pluripotent embryonic stem cells. Proc Natl Acad Sci 100: 11866-11872.

DasGupta R, Fuchs E. 1999. Multiple roles for activated LEF/ TCF transcription complexes during hair follicle development and differentiation. Development 126: 4557-4568.

Dassule HR, Lewis P, Bei M, Maas R, McMahon AP. 2000. Sonic hedgehog regulates growth and morphogenesis of the tooth. Development 127: 4775-4785.

Dhouailly D. 1973. Dermo-epidermal interactions between birds and mammals: Differentiation of cutaneous appendages. J Embryol Exp Morphol 30: 587-603.

Driskell RR, Giangreco A, Jensen KB, Mulder KW, Watt FM. 2009. Sox2-positive dermal papilla cells specify hair follicle type in mammalian epidermis. Development 136: 28152823.

Enshell-Seijffers D, Lindon C, Morgan BA. 2008. The serine protease Corin is a novel modifier of the Agouti pathway. Development 135: 217-225.

Fliniaux I, Mikkola ML, Lefebvre S, Thesleff I. 2008. Identification of $\mathrm{dkk} 4$ as a target of Eda-A1/Edar pathway reveals an unexpected role of ectodysplasin as inhibitor of Wnt signalling in ectodermal placodes. Dev Biol 320: 60-71.

Fuchs E. 2007. Scratching the surface of skin development. Nature 445: 834-842.

Gaide O, Schneider P. 2003. Permanent correction of an inherited ectodermal dysplasia with recombinant EDA. Nat Med 9: 614-618. 
Huh et al.

Harada N, Tamai Y, Ishikawa T, Sauer B, Takaku K, Oshima M, Taketo MM. 1999. Intestinal polyposis in mice with a dominant stable mutation of the $\beta$-catenin gene. EMBO J 18: 5931-5942.

Hardy MH. 1992. The secret life of the hair follicle. Trends Genet 8: 55-61.

Huh SH, Jones J, Warchol ME, Ornitz DM. 2012. Differentiation of the lateral compartment of the cochlea requires a temporally restricted FGF20 signal. PLOS Biol 10: e1001231.

Ito Y, Hamazaki TS, Ohnuma K, Tamaki K, Asashima M, Okochi H. 2007. Isolation of murine hair-inducing cells using the cell surface marker prominin-1/CD133. I Invest Dermatol 127: 1052-1060.

Karlsson L, Bondjers C, Betsholtz C. 1999. Roles for PDGF-A and sonic hedgehog in development of mesenchymal components of the hair follicle. Development 126: 2611-2621.

Kollar EJ. 1970. The induction of hair follicles by embryonic dermal papillae. J Invest Dermatol 55: 374-378.

Kondo S, Miura T. 2010. Reaction-diffusion model as a framework for understanding biological pattern formation. Science 329: 1616-1620.

Laurikkala J, Pispa J, Jung HS, Nieminen P, Mikkola M, Wang X, Saarialho-Kere U, Galceran J, Grosschedl R, Thesleff I. 2002. Regulation of hair follicle development by the TNF signal ectodysplasin and its receptor Edar. Development 129: 25412553.

Lefebvre S, Fliniaux I, Schneider P, Mikkola ML. 2012. Identification of ectodysplasin target genes reveals the involvement of chemokines in hair development. J Invest Dermatol 132: 1094-1102.

Lin CM, Jiang TX, Baker RE, Maini PK, Widelitz RB, Chuong CM. 2009. Spots and stripes: Pleomorphic patterning of stem cells via p-ERK-dependent cell chemotaxis shown by feather morphogenesis and mathematical simulation. Dev Biol 334: 369382.

Mikkola ML, Millar SE. 2006. The mammary bud as a skin appendage: Unique and shared aspects of development. I Mammary Gland Biol Neoplasia 11: 187-203.

Mou C, Jackson B, Schneider P, Overbeek PA, Headon DJ. 2006. Generation of the primary hair follicle pattern. Proc Natl Acad Sci 103: 9075-9080.

Mustonen T, Ilmonen M, Pummila M, Kangas AT, Laurikkala J, Jaatinen R, Pispa J, Gaide O, Schneider P, Thesleff I, et al. 2004. Ectodysplasin A1 promotes placodal cell fate during early morphogenesis of ectodermal appendages. Development 131: 4907-4919.

Närhi K, Jarvinen E, Birchmeier W, Taketo MM, Mikkola ML, Thesleff I. 2008. Sustained epithelial $\beta$-catenin activity induces precocious hair development but disrupts hair follicle down-growth and hair shaft formation. Development 135: 1019-1028.

Närhi K, Tummers M, Ahtiainen L, Itoh N, Thesleff I, Mikkola ML. 2012. Sostdc1 defines the size and number of skin appendage placodes. Dev Biol 364: 149-161.

Ohuchi H, Tao H, Ohata K, Itoh N, Kato S, Noji S, Ono K. 2003. Fibroblast growth factor 10 is required for proper development of the mouse whiskers. Biochem Biophys Res Commun 302: 562-567.

Petiot A, Conti FJ, Grose R, Revest JM, Hodivala-Dilke KM, Dickson C. 2003. A crucial role for Fgfr2-IIIb signalling in epidermal development and hair follicle patterning. Development 130: 5493-5501.

Pummila M, Fliniaux I, Jaatinen R, James MJ, Laurikkala J, Schneider P, Thesleff I, Mikkola ML. 2007. Ectodysplasin has a dual role in ectodermal organogenesis: Inhibition of Bmp activity and induction of Shh expression. Development 134: 117-125.
Richardson GD, Bazzi H, Fantauzzo KA, Waters JM, Crawford H, Hynd P, Christiano AM, Jahoda CA. 2009. KGF and EGF signalling block hair follicle induction and promote interfollicular epidermal fate in developing mouse skin. Development 136: 2153-2164.

Schmidt-Ullrich R, Paus R. 2005. Molecular principles of hair follicle induction and morphogenesis. Bioessays 27: 247-261.

Schmidt-Ullrich R, Tobin DI, Lenhard D, Schneider P, Paus R, Scheidereit C. 2006. NF-кB transmits Eda A1/EdaR signalling to activate Shh and cyclin D1 expression, and controls post-initiation hair placode down growth. Development 133: 1045-1057.

Sennett R, Rendl M. 2012. Mesenchymal-epithelial interactions during hair follicle morphogenesis and cycling. Semin Cell Dev Biol 23: 917-927.

Sick S, Reinker S, Timmer J, Schlake T. 2006. WNT and DKK determine hair follicle spacing through a reaction-diffusion mechanism. Science 314: 1447-1450.

Song HK, Lee SH, Goetinck PF. 2004. FGF-2 signaling is sufficient to induce dermal condensations during feather development. Dev Dyn 231: 741-749.

St-Jacques B, Dassule HR, Karavanova I, Botchkarev VA, Li J, Danielian PS, McMahon JA, Lewis PM, Paus R, McMahon AP. 1998. Sonic hedgehog signaling is essential for hair development. Curr Biol 8: 1058-1068.

Van Exan RJ, Hardy MH. 1980. A spatial relationship between innervation and the early differentiation of vibrissa follicles in the embryonic mouse. J Anat 131: 643-656.

Wells KL, Hadad Y, Ben-Avraham D, Hillel J, Cahaner A, Headon DJ. 2012. Genome-wide SNP scan of pooled DNA reveals nonsense mutation in FGF20 in the Scaleless line of featherless chickens. BMC Genomics 13: 257.

Wong A, Lamothe B, Lee A, Schlessinger J, Lax I. 2002. FRS2 $\alpha$ attenuates FGF receptor signaling by Grb2-mediated recruitment of the ubiquitin ligase Cbl. Proc Natl Acad Sci 99: 6684-6689.

Woo WM, Zhen HH, Oro AE. 2012. Shh maintains dermal papilla identity and hair morphogenesis via a Noggin-Shh regulatory loop. Genes Dev 26: 1235-1246.

Yang CC, Cotsarelis G. 2010. Review of hair follicle dermal cells. J Dermatol Sci 57: 2-11.

Yang J, Meyer M, Muller AK, Bohm F, Grose R, Dauwalder T, Verrey F, Kopf M, Partanen J, Bloch W, et al. 2010. Fibroblast growth factor receptors 1 and 2 in keratinocytes control the epidermal barrier and cutaneous homeostasis. I Cell Biol 188: 935-952.

Zhang Y, Andl T, Yang SH, Teta M, Liu F, Seykora JT, Tobias JW, Piccolo S, Schmidt-Ullrich R, Nagy A, et al. 2008. Activation of $\beta$-catenin signaling programs embryonic epidermis to hair follicle fate. Development 135: 2161-2172.

Zhang Y, Tomann P, Andl T, Gallant NM, Huelsken J, Jerchow B, Birchmeier W, Paus R, Piccolo S, Mikkola ML, et al. 2009. Reciprocal requirements for EDA/EDAR/NF-кB and Wnt/ $\beta$-catenin signaling pathways in hair follicle induction. $\mathrm{DeV}$ Cell 17: 49-61. 


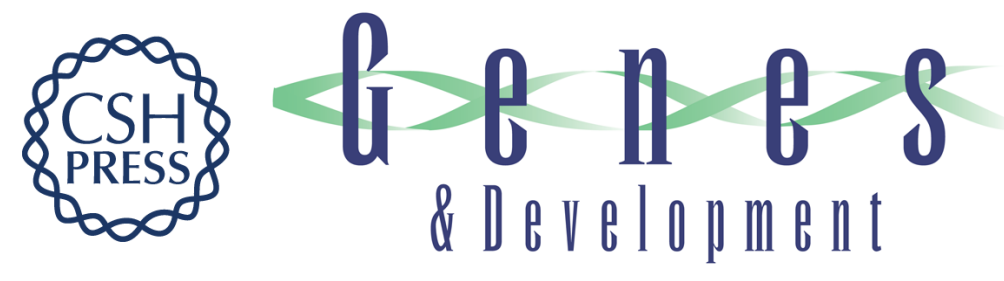

\section{Fgf20 governs formation of primary and secondary dermal condensations in developing hair follicles}

Sung-Ho Huh, Katja Närhi, Päivi H. Lindfors, et al.

Genes Dev. 2013, 27:

Access the most recent version at doi:10.1101/gad.198945.112

Supplemental http://genesdev.cshlp.org/content/suppl/2013/02/21/27.4.450.DC1
Material

References This article cites 47 articles, 23 of which can be accessed free at: http://genesdev.cshlp.org/content/27/4/450.full.html\#ref-list-1

License

Email Alerting Receive free email alerts when new articles cite this article - sign up in the box at the top Service right corner of the article or click here.

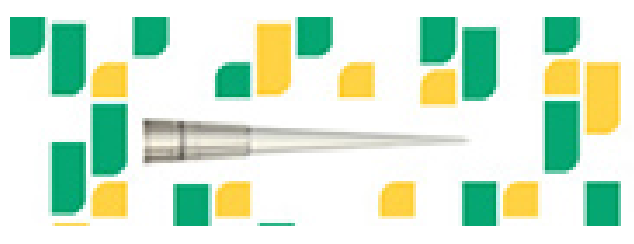

Focused on your science. 